Il en suit que, à la capacité horaire de 4.500 litres la seconde supercentrifugation élimine encore $90 \%$ des bactéries que contenait le lait après le premier traitement, tandis que à la capacité de 6.000 litres, la seconde supercentrifugation élimine $83 \%$.

La double supercentrifugation à l'échelle industrielle de 4.500 litres par heure permet done d'éliminer 99,5\% des bactéries que contenait le lait préchauffé et d'atteindre un effet bactérifuge supérieur à 99, 95\%. Quant à la durée de conservation de ce lait elle est, à $15^{\circ}$, de 8,6 jours en moyenne.

30 décembre 1958.

\title{
BIBLIOGRAPHIE
}

[1] P. Simonart et G. Debeer. The Neth. Milk and Dairy Journal.' 1953, 7,117 .

[2] P. Smonart et G. Debeer. The Neth. Milk and Dairy Journal. 1954, 8, 101.

[3] P. Srmonart et G. Debeer. The Neth. Milk and Dairy Journal. 1954, $9,163$.

[4] P. Simonart, R. Poffe et Wuytack. Le Lait, 1957, 37, 253.

[5] P. Simonart. Document, Fédération Internationale de Laiterie. 1956, 48.

[6] P. Stmonart. Molkerei-Zeitung. 1956, 38, 1303.

\section{RELATION ENTRE LA LACTÉNINE L ET LA LACTOPEROXYDASE}

\author{
par
}

A. PORTMANN et J. E. AUCLAIR

Avec la collaboration technique d'Yvonne GATÉ

Station Centrale de Microbiologie et Recherches Laitières

(Jouy-en-Josas)

\section{I. - INTRODUCTION}

En étudiant l'action inhibitrice du lait cru ou pasteurisé sur les streptocoques lactiques des levains de fromagerie, WRIGHT et Tramer [1] ont montré que, pour certaines souches de streptocoques, l'effet inhibiteur du lait pouvait être attribué à une agglutination des germes par l'agglutinine des globules gras, les cellules bactériennes étant entraînées à la surface du lait avec la crème. Dans un second article [2], ces auteurs ont montré que, pour d'autres souches sensibles, l'inhibition était due vraisemblablement à la lactoperoxydase. Ils trouvaient en effet une étroite corrélation entre la destruction de la lactoperoxydase par divers facteurs physiques ou chimiques (température, $p \mathbf{H}$, inhibiteurs variés) et la disparition de l'inhibition exercée par le lait sur la 
souche Streptococcus cremoris 972 , lorsque le lait servant à cultiver cette souche était soumis aux traitements provoquant la destruction de la lactoperoxydase. WRIGHT et TrAMER montraient en outre que les propriétés de la lactoperoxydase étaient très voisines de celles de la lacténine $\mathrm{L}_{2}$, substance inhibitrice du lait cru particulièrement active sur Str. pyogenes [3], [4], et pensaient que ces deux substances, lactoperoxydase et lacténine $\mathrm{L}_{2}$ étaient identiques.

C'ést cette hypothèse que nous nous sommes proposés de vérifiẹr dans ce travail. Nous avons tout d'abord isolé la lactoperoxydase du lait par adsorption sur résine échangeuse d'ions en dosant à tous les stades de la séparation à la fois l'activité peroxydasique et la tęneur en lacténine $\mathrm{L}_{2}$. Nous avons ensuite comparé l'action du chauffage à différents $p \mathrm{H}$, ainsi que l'effet de l'azide de sodium, sur la destruction de la lactoperoxydase et de la lacténine $\mathrm{L}_{2}$. Enfin nous avons recherché si la peroxydase du raifort (horseradish peroxidase) présentait une activité inhibitrice comparable à celle de la lactoperoxydase.

\section{II. - MÉTHODES}

\section{Préparation de lactoperoxydase brute.}

A partir d'un lait de mélange de plusieurs vaches, nous avons séparé la lactoperoxydase par la méthode de MorRison et al. [5].

Du lactosérum, obtenu par coagulation d'un lait de mélange à l'aide de présure liquide (coagulation en 10 minutes à $40^{\circ}$ C.), était additionné de $1 \%(\mathrm{p} / \mathrm{v})$ de résine échangeuse d'ions IRC-50 (XE-64) préparée suivant la technique décrite par MoRRIson. La peroxydase adsorbée sur la résine était éluée par une solution molaire de $\mathrm{K}_{2} \mathrm{HPO}_{4}$ puis précipitée par $\left(\mathrm{NH}_{4}\right)_{2} \mathrm{SO}_{4}, 4 M$. Le précipité était centrifugé, repris dans du tampon phosphate à $p \mathbf{H} 5,7$, et dialysé contre le même tampon à $6^{\circ} \mathrm{C}$.. La préparation brute de lactoperoxydase ainsi obtenue était environ 150 fois plus active que le lactosérum initial.

\section{Purification de la lactoperoxydase par chromatographie sur colonne.}

La technique de Morrison et al. [5] a été utilisée. On fait passer la solution de peroxydase brute sur une colonne de résine IRC-50 (XE-64), puis on élue successivement avec les tampons phosphate suivants :

- à $p \mathrm{H} 5,7: 0,1 M, 0,2 M$ et $0,4 M$

— à $p \mathrm{H} 7,0: 0,4 M$.

On recueille les éluats par fractions de 5 millilitres au moyen d'un collecteur de fractions automatique. 
La densité optique des fractions obtenues est déterminée à 280,310 et $412 \mathrm{~m} \mu$ à l'aide d'un spectophotomètre Beckman D. U. L'absorption à $280 \mathrm{~m} \mu$ permet d'estimer les protéines totales, l'absorption à $310 \mathrm{~m} \mu$ une protéine rouge mentionnée par MORRISON et al. [5] et par Polis et SHMukLer [6], tandis que l'absorption à $412 \mathrm{~m} \mu$ est due au groupement prosthétique de l'hémoprotéine. Le rapport des densités optiques à 412 et $280 \mathrm{~m} \mu$ est considéré comme un indice de la pureté de la lactoperoxydase [5], [6].

\section{Mesure de l'activité peroxydasique.}

L'activité peroxydasique des diverses préparations était déterminée par la méthode au gaïacol de CHANCE et MAEHLy [7]. Elle était exprimée en unités en prenant comme étalon un échantillon de lait conservé à basse température $\left(-18^{\circ} \mathrm{C}\right.$.). Ce lait étalon contenait par définition 100 unités de peroxydase par millilitre.

\section{Dosage de la lacténine $\mathbf{L}_{2}$.}

Les échantillons de lait destinés à ce dosage étaient préalablement pasteurisés à $63^{\circ} \mathrm{C}$. pendant 30 minutes. Les échantillons de lactosérum étaient stérilisés par filtration sur filtre "Carlson E K S (ou "Ford's sterimats G $\mathrm{S}$ ). Pour les fractions purifiées de lactoperoxydase, nous avons utilisé des membranes filtrantes "Membranfilter Göttingen " no 5, l'enzyme purifié étant retenu par les filtres $\mathrm{E}$ K S et $\mathrm{G} \mathrm{S}$.

Le dosage était effectué suivant la méthode décrite par AuclaIR et HIRsch [3] qui utilise Str. pyogenes (RICHARDs) comme organisme-test. Un échantillon de lait étalon, le même que précédemment, et contenant par définition 100 unités de $\mathrm{L}_{2}$ par millilitre, était utilisé dans chaque dosage à titre de comparaison.

Pour un certain nombre de dosages la souche de Str. cremoris 972 (WRIGHT et TRAMER) a été utilisée comme organisme-test en même temps que Str. pyogenes (RICHARDS). Cette souche 972 a déjà été trouvée particulièrement sensible à la lactoperoxydase [2], [8]. Nous avons toujours obtenu des résultats semblables avec ces deux organismes. L'un et l'autre germes peuvent donc être utilisés indifféremment pour le dosage de $\mathrm{L}_{2}$

\section{Electrophorèse sur papier.}

Nous avons utilisé l'appareil de Machebauf, Dubert et RebexRoTTE (Constructeur Lérès, Paris). L'électrophorèse était faite à la température du laboratoire, pendant quatre à six heures, sous une tension d'environ 10 volts par centimètre en utilisant différents tampons, suivant les préparations à étudier. Les protéines étaient mises en évidence sur les électrophorégrammes par coloration avec 
l'amidoschwarz, suivant la technique de Grassmann et al. [9]. Des courbes d'électrophorèse étaient obtenues à partir des diagrammes ainsi colorés à l'aide d'un photomètre enregistreur automatique Lérès.

A partir de la préparation de lactoperoxydase brute, un isolement des fractions protéiques séparées par électrophorèse sur papier a été réalisé en éluant l'électrophorégramme par bandes parallèles à l'aide d'une technique semblable à celle de GANZIN et Machebeuf [10].

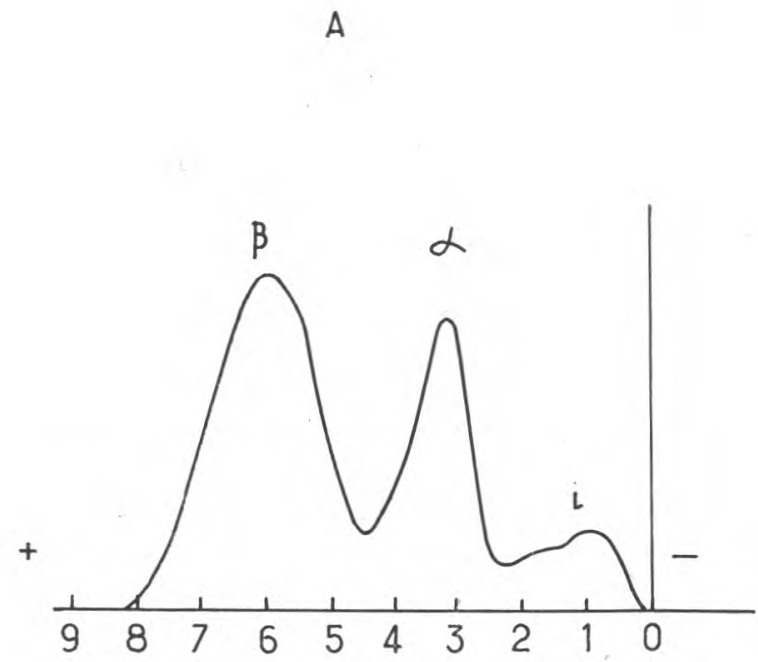

B

A
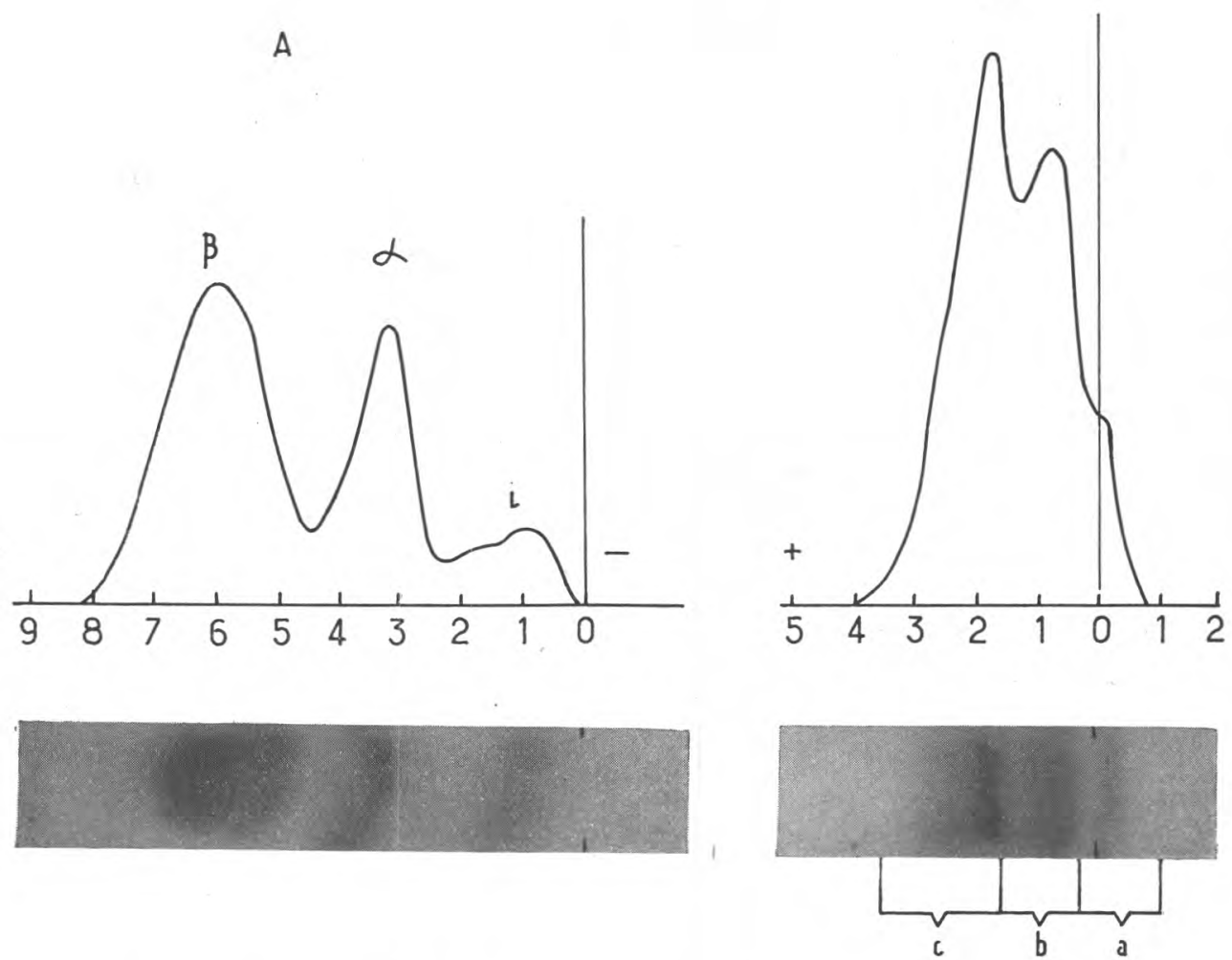

Fig. 1. - A : Electrcphorégramme de lactosérum.

B : Electrophorégramme de lactoperoxydase brute.

Tampon véronal $p \mathrm{H} 9,2 ; 10$ volts/cm., 6 heures. En abcisse, distance parcourue en $\mathrm{cm}$.

$\beta=\beta$-lactoglobuline, $\alpha=\alpha$-lactalbumine, $i=$ immunoglobulines [11]

$a, b$ et $c:$ fractions éluées (voir texte et tableau II). 


\section{III. - RÉSULTATS}

\section{Activité peroxydasique, teneur en lacténine $L_{2}$ et compor- tement électrophorétique de la préparation de lacto- peroxydase brute.}

En partant de lactosérums contenant 60 à 200 unités par millilitre de lactoperoxydase et le même nombre d'unités de lacténine $\mathrm{L}_{2}$, nous avons obtenu des préparations de lactoperoxydase brute dont les teneurs en peroxydase et en lacténine $\mathrm{L}_{2}$ sont indiquées dans le tableau I. On voit que les différentes préparations contiennent 50 à 160 fois les quantités de peroxydase et de $L_{2}$ du lait étalon, et que, pour chaque préparation, les teneurs en peroxydase et en $L_{2}$ sont sensiblement les mêmes.

TABLEAU I

ACTIVITÉ PEROXDASIQUE ET TENEUR EN $\mathbf{L}_{2}$ DE DIFFÉRENTES PREPRATIONS DE LACTOPEROXYDASE BRUTE

\begin{tabular}{|c|c|c|c|}
\hline & $\begin{array}{l}\text { Préparations de } \\
\text { lactoperoxydase brute }\end{array}$ & Lactoperoxydase $(\mathrm{u} / \mathrm{ml}$.) (I) & $\mathrm{L}_{2}(\mathrm{u} / \mathrm{m} 1),(1)$ \\
\hline 1 & $\ldots \ldots \ldots \ldots$ & 6.500 & 6.800 \\
\hline 2 & $\ldots \ldots \ldots \ldots \ldots$ & 8.000 & 12.000 \\
\hline 3 & $\ldots \ldots \ldots \ldots \ldots$ & 15.000 & 16.000 \\
\hline 4 & $\ldots \ldots \ldots \ldots \ldots$ & 5.000 & 5.000 \\
\hline
\end{tabular}

(1) Activité peroxydasique et teneur en $\mathrm{L}_{2}$ rapportées au même lait étalon. Ce lait étalon contient par définition 100 unités par $\mathrm{ml}$. de lactoperoxydase et 100 unités par ml. de lacténine $\mathrm{L}_{2}$.

L'étude électrophorétique d'une de ces préparations de peroxydase brute nous a donné la courbe d'électrophorèse de la figure 1, B. La comparaison avec la courbe d'électrophorèse obtenue avec du lactosérum (figure 1, A) montre qu'une grande partie des protéines du lactosérum ont été éliminées au cours de l'isolement.

L'élution des fractions séparées par électrophorèse sur papier à partir de cette même peroxydase brute (fractions $a, b$ et $c$ de la figure 1, B) nous a donné les résultats indiqués sur le tableau II.

TABLEAU II

ACTIVITÉ PEROXYDASIQUE ET TENEUR EN $L_{2}$ DES FRACTIONS SÉPARÉES PAR ÉLEGTROPHORÉSE SUR PAPIER A PARTIR DE LA PRÉPARATION DE LAGTOPEROXYDASE BRUTE

\begin{tabular}{|c|c|c|}
\hline Fractions (1) & Lactoperoxydase $(\mathrm{u} / \mathrm{ml})(2)$ & $\mathrm{L}_{2}(\mathrm{u} / \mathrm{ml}).(2)$ \\
\hline$a \ldots \ldots \ldots \ldots \ldots$ & traces & 0 \\
\hline$b \ldots \ldots \ldots \ldots \ldots$ & 2.500 & 3.600 \\
\hline$c \ldots \ldots \ldots \ldots \ldots$ & 300 & 230 \\
\hline
\end{tabular}

(1) Voir figure 1, B.

(2) Voir tableau I. 


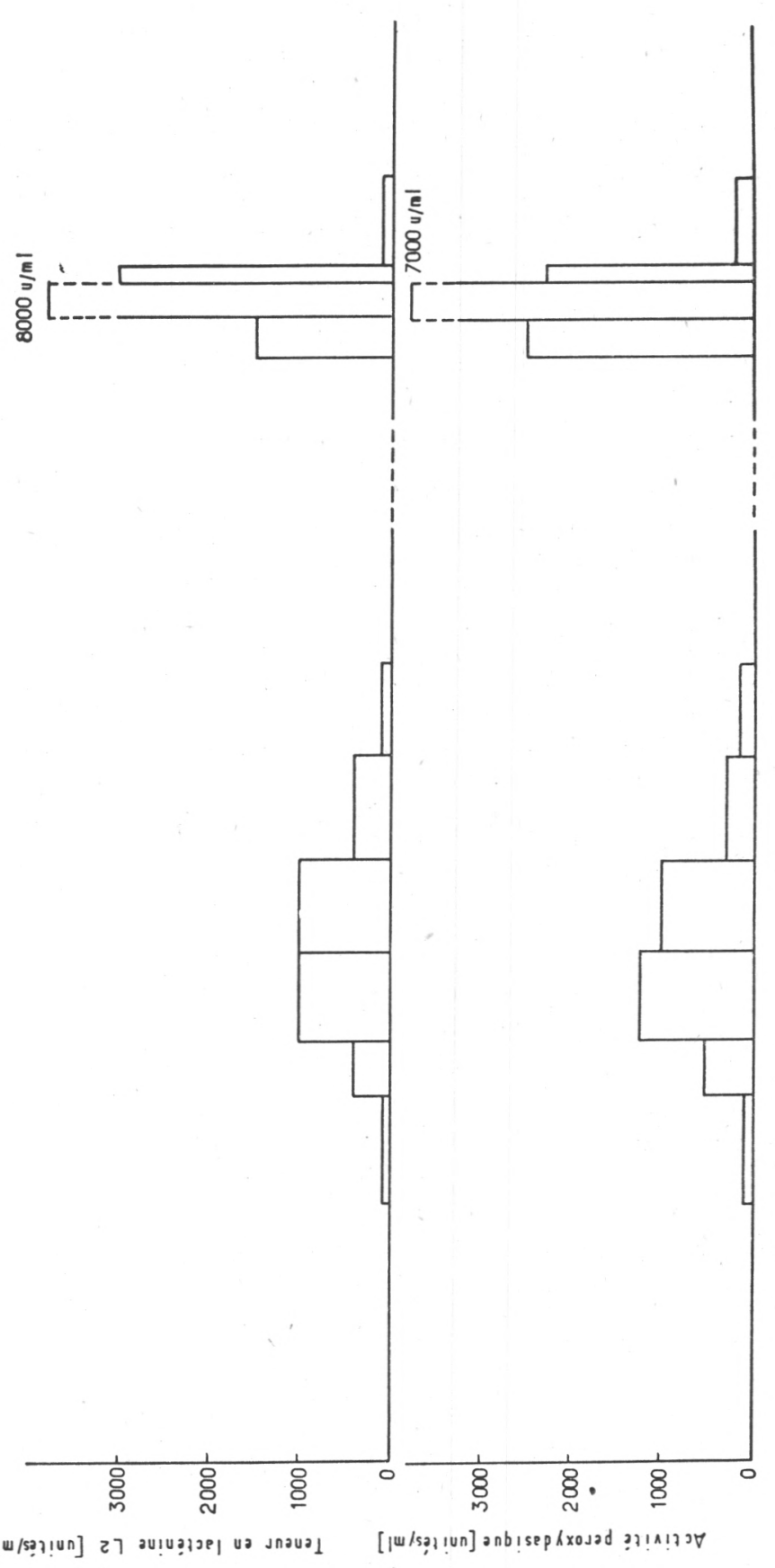




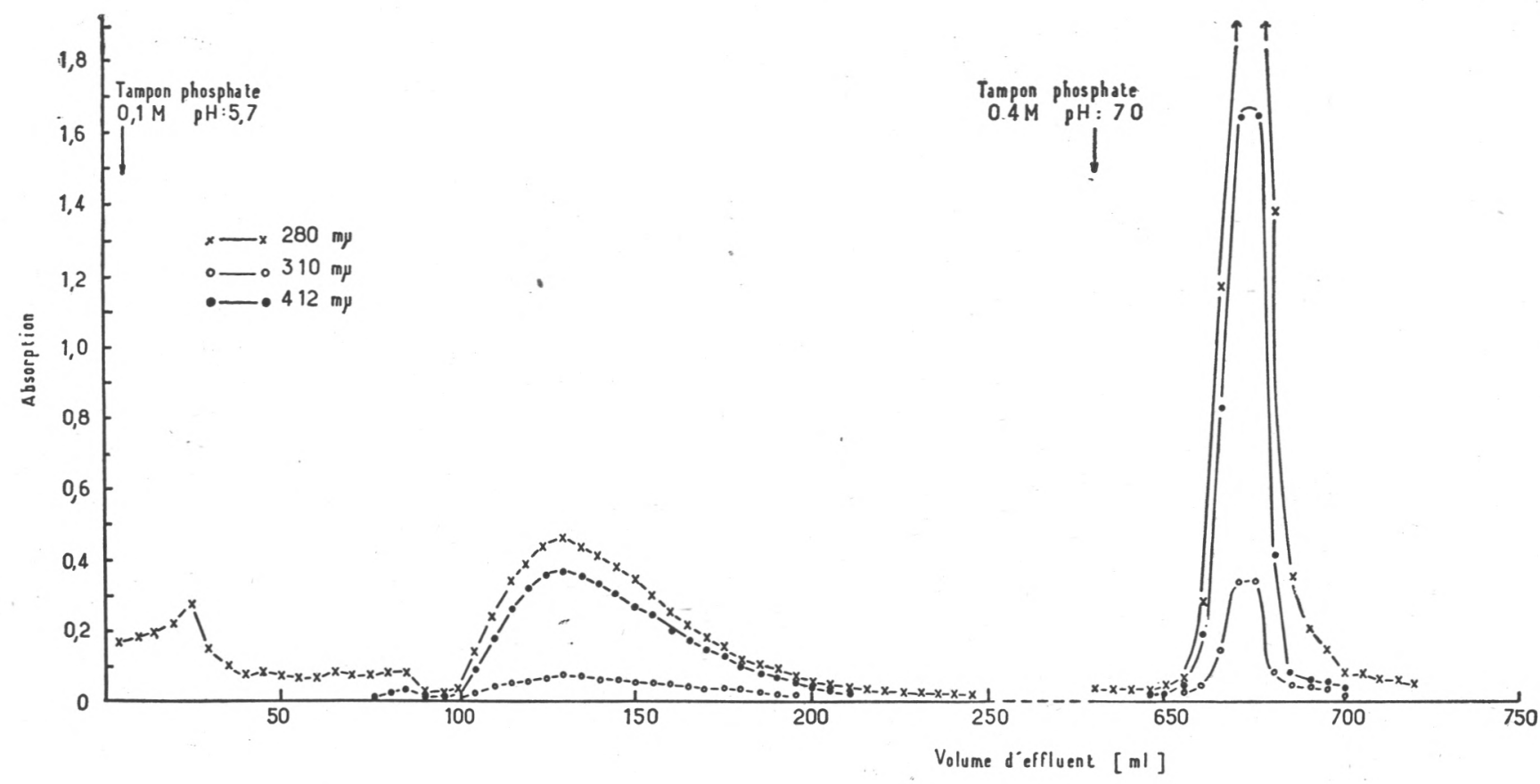

Fig. 2. - Isolement de la lactoperoxydase par chromatographie sur colonne d'Amberlite I R C -50 
On voit que l'activité peroxydasique se retrouve en presque totalité dans la fraction $b$. Cette même fraction contient également la plus grande partie de la lacténine $\mathrm{L}_{2}$. L'activité peroxydasique et la teneur en lacténine $\mathrm{L}_{2}$ des différentes fractions sont encore du même ordre de grandeur.

\section{Activité peroxydasique, teneur en lacténine $L_{2}$ et compor- tement électrophorétique des fractions séparées par chromatographie sur colonne.}

La figure 2 donne l'ensemble des résultats obtenus au cours de cette séparation. On voit que deux fractions protéiques principales sont éluées d'une part par le tampon phosphate $0,1 M$ de $p H 5,7$, d'autre part par le tampon phosphate $0,4 M$ de $p H$ 7,0. Tandis que les densités optiques à 412 et à $280 \mathrm{~m} \mu$ des fractions séparées entre 100 et 250 millilitres d'effluent sont assez voisines (rapport densité optique à $412 \mathrm{~m} \mu$

densité optique à $280 \mathrm{~m}$ voisin de 0,8 ), au contraire, pour les fractions séparées entre 650 et 700 millilitres d'effluent, ce même rapport varie de 0,1 à 0,7 , ce qui montre que ces dernières fractions contiennent, en plus de la lactoperoxydase, une quantité assez importante de protéine étrangère. La figure 2 donne d'autre part l'activité peroxydasique, mesurée par la méthode au gaïacol, ainsi que la teneur en $\mathrm{L}_{2}$ des fractions recueillies au cours de la chromatographie et rassemblées ainsi qu'il est indiqué sur la figure. On constate encore une relation étroite entre l'activité peroxydasique et la teneur en $\mathrm{L}_{2}$ de chaque fraction.

La figure 3 montre par ailleurs les courbes d'électrophorèse obtenues avec les fractions les plus riches en peroxydase rassemblées entre 120 à 200 millilitres d'effluent, et entre 670 et 675 millilitres d'effluent. Dans le premier cas (fig. 3, A) la courbe d'électrophorèse présente un seul pic à 1 centimètre environ du point de départ, tandis que les fractions recueillies entre 670 et 675 millilitres (fig. 3, B donnent une courbe irrégulière indiquant la présence, en plus de la lactoperoxydase (pic à $1 \mathrm{~cm}$.) d'une autre protéine (pic à $2 \mathrm{~cm}$. environ). Ceci confirme les observations indiquées plus haut résultant de la mesure des densités optiques des fractions à 412 et $280 \mathrm{~m} \mu$.

\section{Action du $p H$ et de la température sur la lactoperoxydase et la lacténine $\mathbf{L}_{2}$.}

Les courbes de destruction de la lacténine $\mathrm{L}_{2}$ à des températures et des $p \mathrm{H}$ variables ont été décrites précédemment [3]. Dans les expériences présentes, du lactosérum obtenu comme il a été indiqué plus haut, était stérilisé par filtration sur filtre Carlson E K S, puis additionné de lactoperoxydase brute également sté- 
rilisée par filtration, de façon à obtenir une concentration d'environ 500 unités par millilitre de lactoperoxydase.

Ce lactosérum enrichi en lactoperoxydase était ensuite ajusté avec de la soude $N$ ou de l'acide chlorhydrique $N$ à des $p \mathrm{H}$ variant entre 5 et 9 . Les différentes préparations réparties en tubes de $18 \times 180$ millimètres étaient chauffées pendant 20 minutes à $73^{\circ} \mathrm{C} . \pm 0,1$.

A
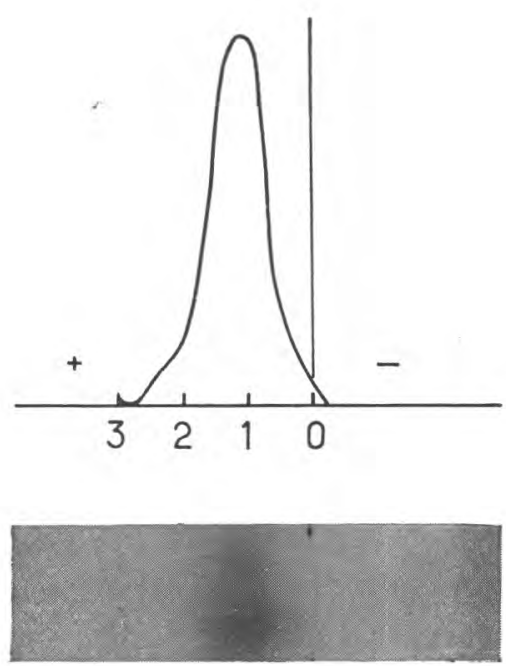

$B$
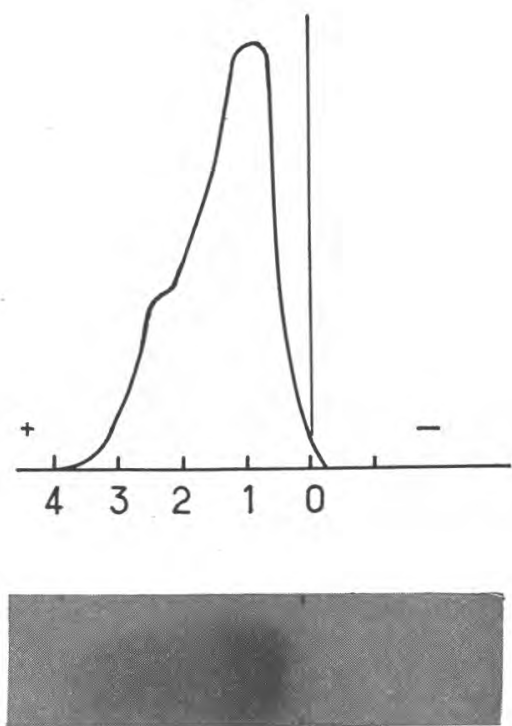

Fig. 3. - A : Electrophorégramme des fractions recueillies par chromatographie entre 120 et $200 \mathrm{ml}$. d'effluent.

B : Electrophorégramme des fractions recueillies entre 670 et $675 \mathrm{ml}$. d'effluent.

Tampon véronal $p \mathrm{H} 9,2 ; 10$ volts/cm.; 6 heures. En abcisse, distance parcourue en $\mathrm{cm}$.

Le tableau III donne les résultats obtenus. On observe encore une relation étroite entre l'activité peroxydasique et la teneur en $\mathrm{L}_{2}$ des diverses préparations.

\section{Action de l'azide de sodium sur la lactoperoxydase et la lacténine $L_{2}$.}

La lactoperoxydase est inactivée par différents inhibiteurs dont l'eau oxygénée et l'azide de sodium. Nous avons déjà montré [4] que la lacténine $\mathrm{L}_{2}$ était également inactivée par l'eau oxygénée. Wright et Tramer [2] ont confirmé ce résultat et observé en outre 
TABLEAU III

AGTIVITÉ PEROXYDASIQUE ET TENEUR EN $L_{2}$ DE LAGTOSÉRUM ENRICHI EN PEROXYDASE ET GHAUFFé A $73^{\circ} \mathrm{C}$ A DES $p H$ VARIABLES

\begin{tabular}{|c|c|c|}
\hline $\begin{array}{c}\text { Lactorésum chauffé à } 73^{\circ} \mathrm{C} . \\
\text { pendant } 20 \text { min. aux } p \mathrm{H} \\
\text { suivants }\end{array}$ & Lactoperoxdase $(\mathrm{u} / \mathrm{ml})(1)$ & $\mathrm{L}_{2}(\mathrm{u} / \mathrm{ml})(1)$ \\
\hline$\ldots \ldots \ldots \ldots \ldots$ & 0 & 0 \\
\hline$\ldots \ldots \ldots \ldots \ldots$ & 190 & 180 \\
\hline$\ldots \ldots \ldots \ldots$ & 250 & 250 \\
\hline$\ldots \ldots \ldots \ldots$ & 250 & 250 \\
\hline$\ldots \ldots \ldots \ldots \ldots$ & 250 & 250 \\
\hline$\ldots \ldots \ldots \ldots \ldots$ & 220 & 200 \\
\hline$\ldots \ldots \ldots \ldots$ & 125 & 125 \\
\hline$\ldots \ldots \ldots \ldots \ldots$ & 80 & 90 \\
\hline$\ldots \ldots \ldots \ldots$ & 0 & 0 \\
\hline Lactosérum non chauffé & 440 & 500 \\
\hline
\end{tabular}

(1) Voir tableau I.

une corrélation entre la destruction de la peroxydase et l'activité de la souche 972 dans du lait traité par l'azide.

Nous avons confirmé à notre tour ce dernier résultat au cours de plusieurs essais dont le tableau IV donne un exemple. On constate

TABLEAU IV

AGTIVITÉ PÉROXYDASIQUE ET TENEUR EN $L_{2}$ DE LACTOSÉRUM ADDITIONNÉ D'AZIDE DE SODIUM

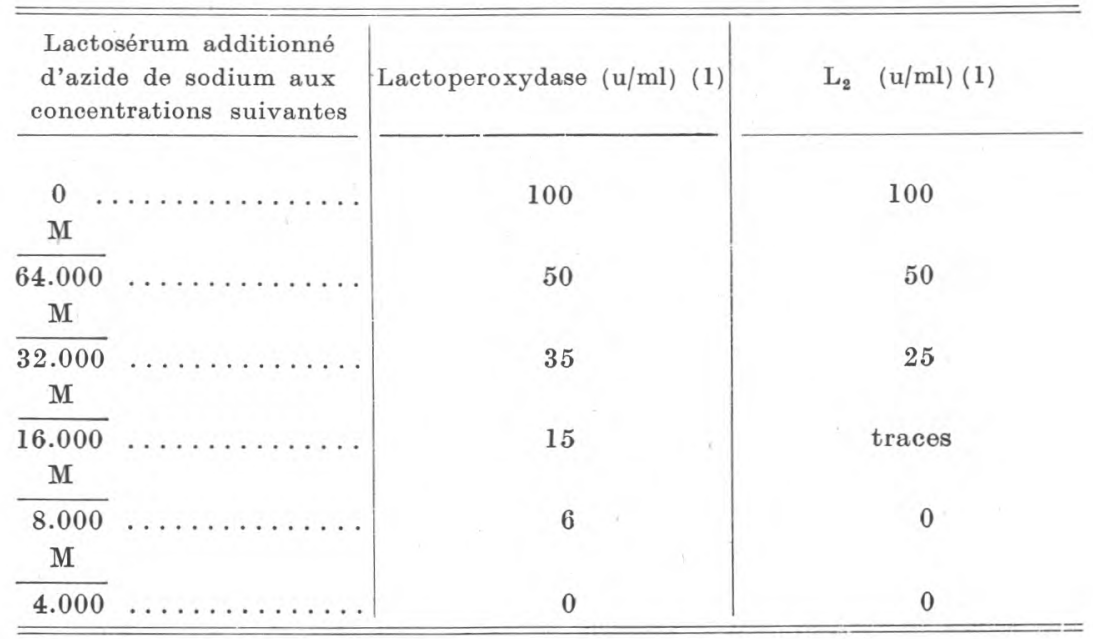

(1) Voir tableau I. 
encore une relation étroite entre l'activité peroxydasique du lactosérum additionnés de différentes concentrations d'azide de sodium et la teneur en lacténine $\mathrm{L}_{2}$ de ces mêmes préparations.

\section{Action inhibitrice de la peroxydase du raifort.}

Une solution de peroxydase de raifort (horse-radish peroxydase, Worthington Biochemical Corp.) a été préparée à raison de 65 milligrammes pour 100 millilitres puis dialysée contre de l'eau distillée. Elle avait une activité peroxydasique de 1.500 unités par millilitres mesurée par la méthode au gaïacol.

Cette préparation ne montra aucune activité inhibitrice par la méthode de dosage de la lacténine $\mathrm{L}_{2}$ soit vis-à-vis de Str. pyogenes, soit vis à vis de Str. cremoris 972 .

\section{IV. - DISCUSSION}

Les résultats précédents confirment l'hypothèse émise par WRIght et TRAMER selon laquelle la lacténine $\mathrm{L}_{2}$ serait identique à la lactoperoxydase.

Nous avons constaté à tous les stades de préparation et de purification de la lactoperoxydase une relation étroite et constante entre l'activité peroxydasique des préparations obtenues et leur teneur en lacténine $\mathrm{L}_{2}$. C'est en particulier le cas des fractions les plus purifiées de lactoperoxydase obtenues par chromatographie, pour lesquelles la lactoperoxydase est dans un grand état de pureté, puisqu'elle représente environ $80 \%$ des protéines totales.

La lacténine $\mathrm{L}_{2}$ disparaît en même temps que l'activité peroxydasique dans les processus qui inactivent la lactoperoxydase : traitement par la chaleur à différents $p \mathrm{H}$ et par l'azide de sodium.

On a done tout lieu de penser que lacténine $\mathrm{L}_{2}$ et lactoperoxydase représentent la même substance.

Mais il est intéressant de noter que l'activité antibactérienne observée sur les deux organismes utilisés, Str. pyogenes et Str. cremoris 972 , est spécifique de la lactoperoxydase et qu'une autre autre peroxydase, telle que la peroxydase du raifort, ne présente pas d'activité inhibitrice vis-à-vis de ces mêmes germes.

\section{RÉSUMÉ}

En isolant la lactoperoxydase du lait par adsorption sur résine échangeuse d'ions, une relation étroite et constante a été trouvée entre l'activité peroxydasique et la teneur en lacténine $\mathrm{L}_{2}$ des préparations obtenues.

La lacténine $\mathrm{L}_{2}$ disparaît en même temps que l'activité peroxydasique au cours des processus qui inactivent la lactoperoxydase tels que le chauffage à différents $p H$ ou l'addition d'azide de sodium. 
Ces résultats confirment l'hypothèse de WRIGHT et Tramer selon laquelle lacténine $\mathrm{L}_{2}$ et lactoperoxydase seraient identiques.

La peroxydase du raifort ne montre aucune activité inhibitrice vis-à-vis des micro-organismes sensibles à la lactoperoxydase tels que Str. pyogenes (RICHARDS) et Str. cremoris 972 .

\section{BIBLIOGRAPHIE}

[1] R. C. Wright et J. Tramer. The influence of eream rising upon the activity of bacteria in heat-treated milk. J. Dairy Res,, 24, 174-183, 1957.

[2] R. C. Wright et J. Tramer. Factors influencing the activity of cheese starters. The role of milk peroxidase. J. Dairy Res., 25, 104-118, 1958.

[3] J. E. Auclair et A. Hirsch. The inhibition of micro-organisms by raw milk. I. The occurence of inhibitory and stimulatory phenomena. Methods of estimation. J. Dairy Res., 20, 45-59, 1953.

[4] J. E. Auclair. The inhibition of micro-organisms by raw milk. III. Distribution and properties of two inhibitory substances, lactenin 1 and lactenin 2. J. Dairy Res., 21, 323-336, 1954.

[5] M. Morrisson, H. B. Hamilton et E. Stotz. The isolation and purification of lactoperoxidase by ion exchange chromatography. J. Biol. Chem., 228, 767-776, 1957.

[6] B. B. Polis et H. W. Shmukler. Crystalline lactoperoxidase. I. Isolation by displacement chromatography. II. Physicochemical and enzymatic properties. J. Biol. Chem., 201, 475-500, 1953.

[7] A. D. Mealhy, in D. Glick. Methods of biochemical analysis, NewYork, 1, 386, 1954.

[8] J. E. Auclair et A. Portmann. Action inhibitrice des lacténines sur les streptocoques lactiques des levains de fromagerie. $X V^{\text {e }}$ Congrès Intern. Laiterie, Londres, 1959 (sous presse).

[9] Ch. Wunderly. Electrophorèse sur papier. Vigot Frères, Paris, p. 40, 1956.

[10] M. Ganzin et M. Machebceuf. Application de la microélectrophorèse sur papier à l'isolement à partir de très petits volumes de sérum de fractions protéiques homogènes à l'électrophorèse et non dénaturées. Bull. Soc. Chim. Biol., 34, 32-42, 1952.

[11] R. Jenness, B. L. Larson, T. L. MoMeekin, A. M. Swanson, C. H. Whitnah et R.Mcl. Whitney, Nomenclature of the proteins of bovine milk. .J. Dairy'Sci., 39, 536-541, 1956. 